\title{
Taxa de mortalidade por neoplasia maligna de mama em mulheres residentes da Região Carbonífera Catarinense no período de 1980 a 2009
}

\author{
Mortality rate of breast cancer among women residents \\ of the Coal Region of Santa Catarina from 1980 to 2009
}

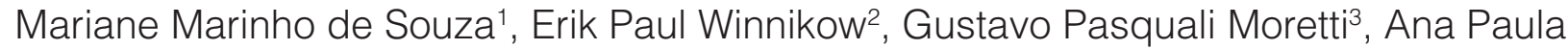 \\ Ronzani Panatto4 ${ }^{4}$ Maria Inês da Rosa5 ${ }^{5}$, Priscyla Waleska Targino de Azevedo Simões ${ }^{6}$
}

\begin{abstract}
Resumo
Introdução: O câncer de mama é o mais prevalente no mundo entre mulheres. Sua incidência no Brasil é em torno de 49 casos para 100.000 mulheres. Objetivo: Estimar o perfil epidemiológico da mortalidade de neoplasia maligna de mama em mulheres residentes da região Carbonífera Catarinense (AMREC) de 1980 a 2009. Metodologia: Estudo temporal, descritivo e ecológico, com dados obtidos do Sistema de Informações sobre Mortalidade e Instituto Brasileiro de Geografia e Estatística, disponíveis pela Secretaria de Estado da Saúde de Santa Catarina. Calculou-se taxa de mortalidade pela divisão do número de óbitos pela população feminina residente no mesmo local e período, e multiplicou-se por 100.000. Resultados: Observou-se maior taxa em Urussanga para todos os anos analisados (9,2/100.000 mulheres) e menor em Treviso, (0,0/100.000 mulheres). Dos 30 anos analisados, 2007 obteve o maior coeficiente médio (13,0/100.000 mulheres), e o menor foi encontrado em 1987 (2,3/100.000 mulheres). Em relação à faixa etária, o maior coeficiente médio foi encontrado nos maiores que 80 anos (63,1/100.000 mulheres). Conclusões: Observamos aumento na taxa de mortalidade por câncer de mama na AMREC, predominantemente em mulheres brancas, casadas, com mais de 80 anos e baixa escolaridade, que pode ser oriundo de características sociodemográficas ou da precocidade dos exames diagnósticos, entre outros. No entanto, futuras pesquisas devem investigar as causas que levaram às estatísticas apresentadas.
\end{abstract}

Palavras-chave: coeficiente de mortalidade; neoplasias da mama; epidemiologia descritiva.

\begin{abstract}
Background: Breast cancer is the most prevalent among women worldwide. Its incidence in Brazil is around 49 cases per 100,000 women. Objective: It was to estimate the epidemiological profile of breast malignancy mortality in women living in the region of the Coal Belt of Santa Catarina (AMREC) from 1980 to 2009. Methods: Study time, with descriptive and ecological data from the Mortality Information System and Instituto Brasileiro de Geografia e Estatística, available by the Ministry of Health of Santa Catarina. We calculated mortality rate by dividing the number of deaths from the female population living in the same place and period, and multiplied by 100,000. Results: There was a higher rate in Urussanga for all years analyzed
\end{abstract}

\footnotetext{
Trabalho realizado no Curso de Medicina da Universidade do Extremo Sul Catarinense (UNESC) - Criciúma (SC), Brasil. 'Bacharel em Medicina pela UNESC - Criciúma (SC), Brasil.

${ }^{2}$ Mestre em Ciências da Saúde pela UNESC - Criciúma (SC); Médico Mastologista especialista pela Sociedade Brasileira de Mastologia - Rio de Janeiro (RJ), Brasil.

${ }^{3}$ Graduando em Ciência da Computação; Pesquisador do Laboratório de Epidemiologia da UNESC - Criciúma (SC), Brasil.

${ }^{4}$ Mestranda em Ciências da Saúde; Bacharel em Farmácia pela UNESC - Criciúma (SC), Brasil.

${ }^{5}$ Doutora em Epidemiologia pela Universidade Federal do Rio Grande do Sul; Médica em Ginecologia pela Universidade Federal de Santa Maria, responsável pelo Laboratório de Epidemiologia da UNESC -Criciúma (SC), Brasil.

${ }^{6}$ Doutora em Ciências da Saúde pela UNESC; Pesquisadora do Laboratório de Epidemiologia da UNESC - Criciúma (SC), Brasil.

Endereço para correspondência: Priscyla Waleska Targino de Azevedo Simões - Avenida Universitária, 1105 - CEP: 88.000-000 - Criciúma (SC), Brasil.

E-mail: pri@unesc.net

Fonte de financiamento: nenhuma.

Conflito de interesses: nada a declarar.
} 
(9.2/100,000 women) and lowest in Treviso (0.0/100,000 women). Of the 30 years analyzed, 2007 had the highest average coefficient (13.0/100,000), and the lowest was found in 1987 (2.3/100,000). In terms of age, the highest average coefficient was found in greater than 80 years $(63.1 / 100,000)$. Conclusions: We found increased mortality rate from breast cancer in AMREC, predominant in white, married, with more than 80 years and low education women that may be coming from sociodemographic characteristics or the precocity of diagnostic tests, among others. However, future research should investigate the causes leading to the statistics presented.

Keywords: mortality rate; breast neoplasms; epidemiology, descriptive.

\section{INTRODUÇÃO}

A mama é uma glândula suscetível a mutações, e o surgimento de uma neoplasia maligna decorre da interação de fatores genéticos e ambientais, dentre os quais se destacam: história familiar, idade superior a 40 anos, sexo feminino, exposição à radiação, nutrição, tabagismo, etilismo, obesidade, primeira menstruação precoce, menopausa tardia (após os 50 anos), primeira gravidez após os 30 anos $^{1,2}$.

A mamografia é o mais importante método de rastreio desse câncer. Sua principal indicação é o rastreamento de rotina, que deve ser feito anualmente, com início a partir dos 40 anos de idade em todas as mulheres sem fatores de risco.

O câncer de mama é uma enfermidade que altera toda a qualidade de vida da paciente, de forma que se entende a importância da presença de uma equipe multidisciplinar para tratá-la integralmente. A escolha do método terapêutico depende de como essa neoplasia se comporta, pois a terapia abrange as modalidades cirúrgica e radioterápica, para tratar lesões regionais, e as modalidades de hormônioterapia e quimioterapia, para manejar o acometimento sistêmico ${ }^{3}$.

A neoplasia maligna de mama é o câncer mais prevalente no mundo entre as mulheres ${ }^{3}$. Nos últimos anos, se observou um aumento na incidência, principalmente em países desenvolvidos, característica essa decorrente da maior disponibilidade de exames mamográficos de rastreamento e pela industrialização das nações ${ }^{4}$. Nesse contexto, nos Estados Unidos da América (EUA), esse carcinoma é o mais comum entre as mulheres, com cerca de 63 novos casos por 100.000 mulheres a cada ano ${ }^{4-7}$.

No Brasil, a neoplasia maligna de mama é considerada a mais prevalente no sexo feminino entre 40 e $69 \operatorname{anos}^{1,8-11}$. A incidência no Brasil encontra-se em torno de 49 casos para cada 100.000 mulheres/ano. Segundo o Instituto Nacional do Câncer (INCA), nos estados brasileiros, esse tumor também apresenta grande representatividade, sendo o mais incidente na Região Sudeste, com 65 novos casos por 100.000 mulheres/ano. Na Região Sul, também é o mais frequente, com incidência de 64/100.000 mulheres/ano (excetuandose os tumores de pele não melanoma $)^{8}$. O estado de Santa Catarina apresenta incidência de aproximadamente 54 novos casos por 100.000 mulheres a cada ano ${ }^{1,8}$.
Dos novos casos que surgem anualmente no mundo cerca de 8,3 por 100.000 mulheres morrem em decorrência dessa doença ${ }^{12}$. Essa neoplasia classifica-se como a quinta causa de morte por câncer em geral, porém é a causa mais frequente de morte por câncer em mulheres ${ }^{12}$.

Nesse contexto, a taxa de mortalidade brasileira continua elevada com aproximadamente 6,6 mortes por 100.000 mulheres anualmente, sendo a primeira causa de morte por neoplasias em mulheres do País ${ }^{1,8,9}$. O estado do Rio de Janeiro é o estado com maior taxa de mortalidade, com 16,9 mortes por 100.000 mulheres/ano, e o estado de Santa Catarina ocupa a quinta posição, com 10 óbitos para cada 100.000 mulheres/ano ${ }^{1,9}$.

Desse modo, este trabalho teve como objetivo avaliar o perfil epidemiológico da mortalidade pela neoplasia maligna de mama em mulheres residentes nas localidades da Associação dos Municípios da Região Carbonífera Catarinense (AMREC) no período de 1980 a 2009.

\section{MÉTODOS}

Esta pesquisa consistiu em um estudo ecológico, descritivo e temporal, que foi aprovado pelo Comitê de Ética e Pesquisa em Seres Humanos da Universidade do Extremo Sul Catarinense sob o protocolo $\mathrm{n}^{\circ} 137 / 2010$.

Utilizamos o capítulo II da décima edição da Classificação Estatística Internacional de Doenças e Problemas Relacionados com a Saúde (CID-10) relacionada aos eventos C50 que representa a neoplasia maligna de mama, e para a nona edição da Classificação Estatística Internacional de Doenças e Problemas Relacionados com a Saúde (CID-9) utilizamos o capítulo II que apresenta o evento 174 representando a neoplasia maligna de mama feminina.

A população foi composta por todos os óbitos de mulheres com neoplasia maligna de mama com registro pelo Sistema de Informações sobre Mortalidade (SIM) no período de 1980 a 2009, residentes nos municípios da Região Carbonífera Catarinense, que é composta por Cocal do Sul, Criciúma, Forquilhinha, Içara, Lauro Muller, Morro da Fumaça, Nova Veneza, Siderópolis, Treviso e Urussanga. 
Os dados populacionais foram referentes aos Censos Demográficos do Instituto Brasileiro de Geografia e Estatística (IBGE) de 1980, 1991, 2000 e 2010. Foram utilizadas estimativas preliminares para os anos intercensitários. O ano de 1996 foi apresentado pela Contagem Populacional realizada pelo IBGE. Os dados relacionados aos anos faltantes foram oriundos de estimativas elaboradas no âmbito do Projeto do Fundo de População das Nações Unidas e IBGE (BRA/4/P31A) População e Desenvolvimento. Os dados populacionais de 2007 a 2009 foram provenientes da Coordenação de População e Indicadores Sociais - IBGE ${ }^{13}$.

Foram construídas séries históricas de 1980 a 2009, período para o qual havia disponibilidade de dados de óbitos no SIM, veiculado pelo Departamento de Informática do Sistema Único de Saúde (DATASUS).

As idades foram estratificadas de acordo com os seguintes grupos adotados pelo DATASUS ${ }^{1}: 20$ a 29, 30 a 39, 40 a 49, 50 a 59, 60 a 69, 70 a 79, 80 anos e mais. A variável grau de instrução foi estratificada de acordo com o tempo de estudo (nunca estudou, 1 a 7 anos, 8 a 11 anos, mais que 12 anos e tempo ignorado), classificação esta atualmente adotada pelo IBGE e Ministério da Saúde ${ }^{13}$.

Em relação à raça/cor dos indivíduos, utilizou-se a seguinte classificação: branca, preta, parda e ignorado. Quanto à variável estado civil, foi analisada de acordo com os seguintes parâmetros: se o indivíduo era solteiro, casado, viúvo, outro e ignorado. Em relação à ocupação, a estratificação foi feita da seguinte maneira: aposentado, dona de casa, trabalhos braçais, e as demais agrupadas em outras ocupações ${ }^{1}$.

Durante a coleta das informações disponibilizadas no SIM, algumas variáveis não foram encontradas em alguns períodos, como a ocupação e raça para os anos de 1980 a 1995.

Calculou-se a frequência absoluta e relativa para o estado civil, raça, grau de instrução e ocupação, e foi calculado o coeficiente bruto de mortalidade pela divisão do número de óbitos pela população feminina residente no mesmo local e período, e multiplicou-se por 100.000 .

Calculamos o coeficiente de mortalidade proporcional e padronizado por faixa etária e município, pois essas variáveis apresentaram dados disponíveis tanto no SIM quanto no IBGE, ambos disponibilizados pelo DATASUS, como também pela Secretaria de Estado da Santa Catarina.

A tabulação dos dados e o acesso ao SIM foram realizados a partir do programa TabNet disponível para acesso on-line no site do DATASUS, e pelo software TabWin versão 3.6. Tanto os indicadores como a análise estatística descritiva foram desenvolvidos no software Excel versão 2007.

\section{RESULTADOS}

Durante o período de 1980 a 2009, ocorreram 321 óbitos por câncer de mama nos municípios da AMREC. O coeficiente bruto médio dos anos analisados foi de 6,9/100.000 mulheres, e o perfil descritivo da população estudada encontra-se disponível na Tabela 1.

Os municípios de Urussanga e Criciúma apresentaram para todo o período os maiores coeficientes médios, 9,2/100.000 mulheres e 8,1/100.000 mulheres, respectivamente. O município de Treviso foi o que apresentou o menor coeficiente médio para todos os anos, 0,0/100.000 mulheres.

De todos os 30 anos analisados, 2007 obteve o maior coeficiente médio (13,0/100.000 mulheres), e o menor foi encontrado em 1987 (2,3/100.000 mulheres).

Em relação à faixa etária, observou-se um aumento progressivo do coeficiente médio do primeiro grupo (de 20 a 29 anos) para o último (80 anos e mais). O maior coeficiente médio foi encontrado nos maiores que 80 anos 63,1/100.000 mulheres —, seguindo-se das faixas etárias de 70 a 79 anos $-39,0 / 100.000$ mulheres - , e o menor na faixa etária dos 20 a 29 anos - coeficiente médio de mortalidade de 0,4/100.000 mulheres.

Em relação ao grau de instrução, os indivíduos que estudaram de 1 a 7 anos apresentaram o maior coeficiente médio (5,8/100.000 mulheres), e o menor coeficiente médio foi obtido nos que estudaram 12 anos ou mais $(0,2 / 100.000$ mulheres).

Com relação ao estado civil, as mulheres casadas foram as que obtiveram a maior taxa de mortalidade média (6,2/100.000 mulheres), seguindo-se das viúvas com 2,2/100.000 mulheres e solteiras com 1,6/100.000 mulheres.

A ocupação de dona de casa foi a que apresentou o maior coeficiente médio, com média de 4,3/100.000 mulheres, seguida dos trabalhos braçais, com média de 3,4/100.000 mulheres, e da ocupação aposentados/pensionistas, com média de 2,7/100.000 mulheres.

Sobre as diferentes raças, observou-se uma predominância da raça branca, com o coeficiente médio de 11,2/100.000 mulheres, seguida da raça negra com 0,4/100.000 mulheres, e logo após pela raça parda com $0,1 / 100.000$ mulheres.

\section{DISCUSSÃO}

O conhecimento dos dados epidemiológicos do câncer de mama de uma determinada região permite uma reflexão da importância dessa neoplasia, que só no ano de 2008 representou aproximadamente $23 \%$ dos novos casos de neoplasias malignas no mundo ${ }^{12}$. 
Tabela 1. Características da amostra

\begin{tabular}{|c|c|c|c|c|c|c|}
\hline \multirow[b]{2}{*}{ Variável } & \multicolumn{6}{|c|}{ Ano $n(\%) n=321$} \\
\hline & $\begin{array}{c}1980-1984 \\
n=31\end{array}$ & $\begin{array}{c}1985-1989 \\
n=27\end{array}$ & $\begin{array}{c}1990-1994 \\
n=42\end{array}$ & $\begin{array}{c}1995-1999 \\
n=71\end{array}$ & $\begin{array}{c}2000-2004 \\
n=60\end{array}$ & $\begin{array}{c}2005-2009 \\
n=90\end{array}$ \\
\hline \multicolumn{7}{|l|}{ Municípios } \\
\hline Cocal do Sul & $0(0,0)$ & $0(0,0)$ & $0(0,0)$ & $3(4,2)$ & $0(0,0)$ & $6(6,7)$ \\
\hline Criciúma & $22(71,0)$ & $19(70,4)$ & $21(50,0)$ & $46(64,8)$ & $35(58,3)$ & $50(55,6)$ \\
\hline Forquilhinha & $0(0,0)$ & $0(0,0)$ & $0(0,0)$ & $5(7,0)$ & $5(8,3)$ & $5(5,6)$ \\
\hline Içara & $3(9,7)$ & $3(11,1)$ & $6(14,3)$ & $3(4,2)$ & $6(10,0)$ & $13(14,4)$ \\
\hline Lauro Muller & $1(3,2)$ & $2(7,4)$ & $1(2,4)$ & $1(1,4)$ & $5(8,3)$ & $0(0,0)$ \\
\hline M. da Fumaça & $0(0,0)$ & $0(0,0)$ & $2(4,8)$ & $2(2,8)$ & $3(5,0)$ & $6(6,7)$ \\
\hline Nova Veneza & $1(3,2)$ & $1(3,7)$ & $2(4,8)$ & $3(4,2)$ & $0(0,0)$ & $2(2,2)$ \\
\hline Siderópolis & $2(6,5)$ & $0(0,0)$ & $2(4,8)$ & $2(2,8)$ & $0(0,0)$ & $3(3,3)$ \\
\hline Treviso & $0(0,0)$ & $0(0,0)$ & $0(0,0)$ & $0(0,0)$ & $0(0,0)$ & $0(0,0)$ \\
\hline Urussanga & $2(6,5)$ & $2(7,4)$ & $8(19,0)$ & $6(8,5)$ & $6(10,0)$ & $5(5,6)$ \\
\hline \multicolumn{7}{|l|}{ Faixa etária } \\
\hline $20-29$ & $0(0,0)$ & $0(0,0)$ & $1(2,4)$ & $1(1,4)$ & $1(1,7)$ & $0(0,0)$ \\
\hline $30-39$ & $6(19,4)$ & $3(11,1)$ & $4(9,5)$ & $9(12,7)$ & $6(10,0)$ & $9(10,0)$ \\
\hline $40-49$ & $8(25,8)$ & $5(18,5)$ & $10(23,8)$ & $19(26,8)$ & $10(16,7)$ & $16(17,8)$ \\
\hline $50-59$ & $8(25,8)$ & $9(33,3)$ & $9(21,4)$ & $17(23,9)$ & $21(35,0)$ & $32(35,6)$ \\
\hline $60-69$ & $3(9,7)$ & $8(29,6)$ & $10(23,8)$ & $14(19,7)$ & $9(15,0)$ & $17(18,9)$ \\
\hline $70-79$ & $3(9,7)$ & $1(3,7)$ & $7(16,7)$ & $9(12,7)$ & $6(10,0)$ & $11(12,2)$ \\
\hline $80+$ & $3(9,7)$ & $1(3,7)$ & $1(2,4)$ & $2(2,8)$ & $7(11,7)$ & $5(5,6)$ \\
\hline \multicolumn{7}{|l|}{ Escolaridade } \\
\hline Nenhuma & $5(16,1)$ & $3(11,1)$ & $7(16,7)$ & $3(4,2)$ & $6(10,0)$ & $2(2,2)$ \\
\hline 1 a 7 anos & $20(64,5)$ & $18(66,7)$ & $22(52,4)$ & $14(19,7)$ & $37(61,7)$ & $62(68,9)$ \\
\hline 8 a 11 anos & $1(3,2)$ & $2(7,4)$ & $5(11,9)$ & $3(4,2)$ & $3(5,0)$ & $9(10,0)$ \\
\hline $12+$ & $0(0,0)$ & $1(3,7)$ & $0(0,0)$ & $0(0,0)$ & $1(1,7)$ & $5(5,6)$ \\
\hline Ignorado & $5(16,1)$ & $3(11,1)$ & $8(19,0)$ & $51(71,8)$ & $13(21,7)$ & $12(13,3)$ \\
\hline \multicolumn{7}{|l|}{ Estado civil } \\
\hline Solteiro & $7(22,6)$ & $3(11,1)$ & $13(31,0)$ & $9(12,7)$ & $9(15,0)$ & $6(6,7)$ \\
\hline Casado & $19(61,3)$ & $16(59,3)$ & $18(42,9)$ & $44(62,0)$ & $31(51,7)$ & $58(64,4)$ \\
\hline Viúvo & $4(12,9)$ & $8(29,6)$ & $10(23,8)$ & $12(16,9)$ & $15(25,0)$ & $18(20,0)$ \\
\hline Outro & $1(3,2)$ & $0(0,0)$ & $0(0,0)$ & $2(2,8)$ & $0(0,0)$ & $7(7,8)$ \\
\hline Ignorado & $0(0,0)$ & $0(0,0)$ & $1(2,4)$ & $4(5,6)$ & $5(8,3)$ & $1(1,1)$ \\
\hline Ocupação & & & & 1996-1999 & & \\
\hline Aposentado & - & - & - & $21(36,2)$ & $15(25,0)$ & $1(1,1)$ \\
\hline Dona de casa & - & - & - & $28(48,3)$ & $26(43,3)$ & $6(6,7)$ \\
\hline Outros & - & - & - & $7(12,1)$ & $12(20,0)$ & $7(7,8)$ \\
\hline Outros trabalhos braçais & - & - & - & $0(0,0)$ & $1(1,7)$ & $47(52,2)$ \\
\hline Ignorado & - & - & - & $2(3,4)$ & $6(10,0)$ & $29(32,2)$ \\
\hline Raça/cor & & & & 1996-1999 & & \\
\hline Branca & - & - & - & $17(29,3)$ & $56(93,3)$ & $84(93,3)$ \\
\hline Preta & - & - & - & $1(1,7)$ & $2(3,3)$ & $3(3,3)$ \\
\hline Parda & - & - & - & $0(0,0)$ & $0(0,0)$ & $1(1,1)$ \\
\hline Ignorado & - & - & - & $40(69,0)$ & $2(3,3)$ & $2(2,2)$ \\
\hline
\end{tabular}


Os dados epidemiológicos da mortalidade pela neoplasia de mama da AMREC descritos neste estudo revelaram uma mortalidade superior nesta região quando comparada com a mortalidade do estado de Santa Catarina, que é de 5,6/100.000 mulheres, bem como com as taxas da Região Sul do Brasil, que apresenta um coeficiente médio de mortalidade de 6,8/100.000 mulheres, e no Brasil com 5,3/100.000 mulheres $^{13-15}$. Um estudo europeu realizado em 1999 sobre a população mundial demonstrou o coeficiente médio de mortalidade para a América do Norte de 10,7/100.000 mulheres, a América do Sul (tropical) de 4,0/100.000 mulheres, a América do Sul (temperada) de 1,3/100.000 mulheres e o leste europeu de 11,4/100.000 mulheres ${ }^{16}$. Essas diferenças podem ser explicadas em razão da notificação dos óbitos, que nos países desenvolvidos podem ter um controle mais rigoroso, aumentando assim os registros de óbitos descritos nesses locais.

O município de Treviso apresentou dados míninos do coeficiente de mortalidade referente a todos os anos estudados - 0,0/100.000 mulheres. Tal resultado pode ser explicado pelo fato de que boa parte da população desse município vai fazer tratamento nas cidades vizinhas e, consequentemente, diminui a notificação referente ao município em que o indivíduo realmente reside.

Foi observada uma tendência de mortalidade por neoplasia maligna de mama nos municípios da AMREC no período compreendido entre 1980 e 2009, especialmente de 2006 a 2009, estando em concordância com os números referentes à Região Sul, ao Brasil (obtidos via SIM) e a alguns países em desenvolvimento, de acordo com o relatório apresentado pelo GLOBOCAN $^{13,16}$. Uma importante observação que pode ser feita em relação à mortalidade por câncer de mama no mundo é que em alguns países desenvolvidos que dispõem de programas eficientes de rastreamento há um declínio nas taxas de mortalidade, principalmente a partir do final da década de $1980^{12,17-19}$. É importante ressaltar que o Brasil tem um programa de rastreamento do câncer de mama, porém ainda não é amplamente difundido e utilizado por todo o País, o que por si só justifica o aumento da mortalidade por esse tipo de câncer no nosso País. Existem iniciativas isoladas de programas de rastreamento, como o ocorrido em Barretos entre 2003 e 2005, em que 17.964 mulheres foram examinadas por mamografia, e ficou comprovada a relevância desse exame para a detecção precoce dessa doença ${ }^{18}$. Essa iniciativa prova que é possível realizar um programa de rastreamento em um país em desenvolvimento, e o reflexo será o mesmo observado nos países que já o fazem, ou seja, uma redução na mortalidade.

Os maiores coeficientes médios de mortalidade foram encontrados na população com mais de 80 anos $(63,1 / 100.000$ mulheres), representando cerca de 6,3\% do total de óbitos, porém o grupo entre 50 a 59 anos também apresenta um número de óbitos expressivo, com um coeficiente médio de mortalidade de 30,6/100.000 mulheres, representando 31,7\% das mortes em decorrência de câncer de mama. Nossos dados estão em concordância com países desenvolvidos e em desenvolvimento, porém em um estudo de base populacional sobre a mortalidade nos EUA revelou um leve declínio na mortalidade em todas as faixas etárias, com queda acentuada na idade de 55 a 73 anos $^{20,21}$. $\mathrm{O}$ aumento da mortalidade com o passar dos anos pode ser explicado pelo fato de que muitas vezes indivíduos mais velhos tendem a diminuir a frequência dos exames de rotina necessários para manter uma boa saúde e, consequentemente, tornamse mais vulneráveis à descoberta de tumores em estágios mais avançados. Nas faixas etárias mais avançadas, a letalidade da doença é maior, em razão não só da possibilidade de diagnóstico tardio mas também dos próprios fatores fisiológicos e das comorbidades mais frequentes na população idosa.

Outra variável analisada em nosso estudo foi o grau de instrução, representado pelo tempo de estudo, que apresentou uma maior mortalidade em indivíduos com 1 a 7 anos de estudo (53,9\% dos óbitos) e um índice menor nos que tiveram 12 ou mais anos de estudo (2,2\% dos óbitos). Nossos achados estão de acordo com uma coorte realizada na cidade de São Paulo no período de 1994 a 1995; segundo esse mesmo trabalho, pacientes com 12 anos ou mais anos de estudo estavam associados à mortalidade pela doença em estágio inicial ${ }^{22}$. Esses dados podem sugerir que uma maior escolaridade esteja associada ao maior conhecimento sobre saúde, com destaque à prevenção e diagnóstico precoce da doença.

Em relação à raça, identificamos $75,5 \%$ dos óbitos em indivíduos de raça branca, o que pode ser explicado pela composição étnica da AMREC, que na sua grande maioria é composta por caucasianos. Como não temos o conhecimento da mortalidade por câncer de mama nas diferentes raças, ainda não é possível inferir se a taxa de mortalidade é diferente entre os grupos raciais. No entanto, alguns estudos demonstram que a raça negra apresenta maior mortalidade de câncer de mama em relação à raça branca, e, além disso, também existem estudos norte-americanos e mundiais que demonstram um declínio na mortalidade na raça branca ${ }^{23-25}$.

Considerando os resultados apresentados, pode-se concluir que o câncer de mama é a neoplasia mais frequente no sexo feminino e quando detectada precocemente apresenta menores taxas de mortalidade.

Assim como outros países em desenvolvimento ${ }^{12}$, na região da AMREC, se observou um aumento na taxa de mortalidade por câncer de mama na maior parte do período analisado.

Em relação à mortalidade por faixa etária, observou-se que o grupo de indivíduos com mais de 80 anos foi o grupo 
com as maiores taxas de mortalidade, sendo condizente com os dados mundiais.

A população predominante de indivíduos com câncer de mama nos residentes da Região Carbonífera Catarinense foi composta por mulheres brancas, casadas, de meia idade, com baixo grau de instrução e donas de casa.

A neoplasia maligna de mama apresenta fatores de risco que não são passíveis de mudança (genéticos) e outros que podem mudar de acordo com o comportamento do indivíduo (alimentação, obesidade, etilismo). Visto isso, fica clara a importância do esclarecimento da população quanto à necessidade de evitar alguns fatores de risco (obesidade, etilismo, tabagismo) e de iniciar o rastreamento da doença aos 40 anos de idade.

A Região Carbonífera Catarinense, local de origem dos dados utilizados em nossa pesquisa, é constituída de várias minas de carvão, algumas ativas durante o período do estudo. Como é sabido, o carvão pode estar associado ao câncer de mama; no entanto, nosso estudo não buscou verificar essa associação, sendo sugestão que futuros estudos sejam realizados na região acima citada buscando avaliar essa questão.

Este estudo apresentou um viés na análise da ocupação, pois o SIM não apresentou essa informação para o período de 1980 a 1995. Em relação à raça/cor, o SIM também não apresentou essa variável para os anos de 1980 a 1995. Essa falta de dados pode ser explicada por divergências entre a metodologia de coleta dos dados referente ao CID-9 e ao CID-10. Nosso estudo também apresentou uma possível limitação pelo uso de dados secundários e pela falta de completitude de informações no SIM, especialmente para as décadas de 1980 e 1990. Assim, este estudo apresentou um viés (de informação) na análise da ocupação.

\section{CONCLUSÃO}

De acordo com nosso estudo, em geral, houve um aumento da mortalidade em decorrência do câncer de mama na AMREC no período de 1980 a 2009, predominante em mulheres brancas, casadas, com mais de 80 anos e baixa escolaridade.

Concluímos também que a taxa média da AMREC foi superior à Região Sul brasileira e ao Brasil, apesar de ser a sexta menor taxa de mortalidade do estado de Santa Catarina. Mesmo apresentando uma das menores taxas do estado, novos questionamentos surgem, com destaque aos motivos desse aumento geral. Por se tratar de um estudo ecológico, futuras pesquisas devem investigar as causas que resultaram nas estatísticas apresentadas em nosso estudo.

\section{REFERÊNCIAS}

1. Ziegler RG, Hoover RN, Pike MC, Hildesheim A, Nomura AM, West DW, et al. Migration patterns and breast cancer risk in Asian-American women. J Natl Cancer Inst. 1993;85(22):1819-27.

2. Peto J, Houlston RS. Genetics and common cancer. Eur J Cancer. 2001;37(Suppl 8):S88-96.

3. Coelho JM, Vianna LL, Silva HMS. Propedêutica em Mastologia. Rio de Janeiro: Guanabara Koogan; 2005. 549 p.

4. Peled R, Carmil D, Siboni-Samocha O, Shoham-Vardi I. Breast cancer, psychological distress and life events among young women. BMC Cancer. 2008;8:245.

5. Siegel R, Naishadham D, Jemal A. Cancer statistics. CA Cancer J Clin. 2012;62(1):10-29.

6. Ravdin PM, Cronin KA, Howlader N, Berg CD, Chlebowski RT, Feuer EJ, et al. The decrease in breast-cancer incidence in 2003 in the United States. N Engl J Med. 2007;356(16):1670-4.

7. Partridge AH, Winer EP. On mammography-more agreement than disagreement. N Engl J Med. 2009;361(26):2499-501.

8. INCA [Internet]. Câncer - tipo - mama. 2012. [Cited 2012 Jun. 05]. Available from: http://www2.inca.gov.br/wps/wcm/connect/ tiposdecancer/site/home/mama

9. Crippa CG. Perfil clínico e epidemiológico do câncer de mama em mulheres jovens. ACM. 2005;32:50-8.
10. Kolling FL, Santos JS. A influência dos fatores de risco nutricionais no desenvolvimento de câncer de mama em pacientes ambulatoriais do interior do Rio Grande do Sul, Brasil. Sci Med. 2009;19:115-21.

11. Gonçalves LLC, de Lima AV, Brito SE, de Oliveira MM, de Oliveira LRA, Abud ACF, et al. Mulheres portadoras de câncer de mama: conhecimento e acesso às medidas de detecção precoce. Rev Enferm. 2009;17:362-7.

12. WHO. Breast cancer incidence and mortality worldwide in 2008 Summary [Internet]. GLOBOCAN: World Health Organization. [cited 2011 mai. 17]. Available from: http:/globocan.iarc.fr/factsheets/ cancers/breast.asp

13. DATASUS. Mortalidade - Notas técnicas [Internet]. BRASIL: Sistema de Informações Sobre Mortalidade. Ministério da Saúde. [cited 2011 maio. 17]. Available from: http://tabnet.datasus.gov.br/cgi/sim/obt10descr.htm

14. IBGE. Ministério do Planejamento, Orçamento e Gestão [Internet]. BRASIL: Instituto Brasileiro de Geografia e Estatística. Ministério do Planejamento, Orçamento e Gestão. [cited 17 mai. 2011]. Available from: http://www.ibge.gov.br/home/estatistica/populacao/censo2000/ universo.php?tipo=31\&paginaatual $=1 \& u f=42 \&$ letra $=\mathrm{A}$

15. Stankov S, Stankov K. Descriptive epidemiology of breast cancer in Vojvodina. Breast. 2011;20(2):192-5.

16. Max P, Paola P. Global Cancer Statistics. CA Cancer J Clin. 1999;49:33-6 4.

17. Jemal A, Siegel R, Ward E, Hao Y, Xu J, Thun MJ. Cancer statistics, 2009. CA Cancer J Clin. 2009;59(4):225-49. 
18. Boyle P. Breast cancer control: signs of progress, but more work required. Breast. 2005;14(6):429-38.

19. Haikel J, Raphael L. Avaliação inicial de um programa de detecção precoce do câncer de mama, por meio de mamografia, na região de Barretos [tese de doutorado]. São Paulo: Universidade de São Paulo; 2010.

20. Louwman WJ, Vulto JC, Verhoeven RH, Nieuwenhuijzen GA, Coebergh JW, Voogd AC. Clinical epidemiology of breast cancer in the elderly. Eur J Cancer. 2007;43(15):2242-52.

21. Wakai K, Suzuki S, Ohno Y, Kawamura T, Tamakoshi A, Aoki R. Epidemiology of breast cancer in Japan. Int J Epidemiol. 1995;24(2):285-91.

22. Ana GCO, Snitcovsky IML. Influence of socioeconomic status and education level in the prognosis of breast cancer patients. ACR. 2010;30:240-4.
23. Yasmeen F, Hyndman RJ, Erbas B. Forecasting age-related changes in breast cancer mortality among white and black US women: a functional data approach. Cancer Epidemiol. 2010;34(5):542-9.

24. Barlow WE, Lehman CD, Zheng Y, Ballard-Barbash R, Yankaskas BC, Cutter GR, et al. Performance of diagnostic mammography for women with signs or symptoms of breast cancer. J Natl Cancer Inst. 2002;94(15):1151-9.

25. Tian N, Goovaerts P, Zhan FB, Wilson JG. Identification of racial disparities in breast cancer mortality: does scale matter? Int J Health Geogr. 2010;9:35.

Recebido em: 17/12/2012 Aprovado em: 25/11/2013 